\title{
Spin Description in the Star Product and the Path Integral Formalism
}

\author{
S. Odendahl* and P. Henselder ${ }^{\dagger}$ \\ Fachbereich Physik, Universität Dortmund \\ 44221 Dortmund
}

February 23, 2020

\begin{abstract}
The spin can be described in the star product formalism by extending the bosonic Moyal product in the fermionic sector. The fermionic star product is then the Clifford product of geometric algebra and it is possible to formulate the fermionic star product formalism in analogy to the bosonic star product formalism. For the fermionic star product description of spin, one can then establish the relation to other approaches that describe spin with fermionic variables, i.e. the operator formalism and the path integral formalism. It is shown that the fermionic star product formalism and the fermionic path integral formalism are related in analogy to their bosonic counterparts.
\end{abstract}

\section{Introduction}

The star product formalism as it was established by Bayen et al. in 1 gives an alternative description of quantum mechanics on the phase space [2. One might then wonder if it is also possible to describe spin in the star product formalism. Berezin and Marinov showed in [3] how the spin can be described in the context of pseudoclassical mechanics. So it appears natural to apply the program of deformation quantization to pseudoclassical mechanics in order to obtain a description of spin with a fermionic star product [4. With the fermionic star product it is then possible to find in analogy to the bosonic star product formalism spin Wigner functions as eigenfunctions of a fermionic star eigenvalue equation and a spin star exponential that describes the time development.

A fermionic star product already appeared in [1, whereas a systematic account to a fermionic star product formalism was given in 5. With the fermionic star product the Grassmann algebra is deformed into a Clifford algebra, so that such a Clifford star product can be interpreted as the geometric product of geometric algebra in a superanalytic formulation [6. Geometric algebra goes back to early ideas of Hamilton, Grassmann, and Clifford and was developed into a full formalism by Hestenes in [7] and [8]. From then on it was applied to a wide range of physical problems and in particular it was used for the description of

\footnotetext{
*sven.odendahl@uni-dortmund.de

${ }_{\dagger}^{\dagger}$ peter.henselder@uni-dortmund.de
} 
spin (for a thorough discussion of geometric algebra see [9]). The superanalytic formulation of geometric algebra and its relation to pseudoclassical mechanics was established in [10] so that - in conjunction with the fermionic Clifford star product - a natural geometric interpretation of the fermionic star product formalism is obtained. Furthermore, it now appears natural to combine the fermionic and the bosonic star product formalism (see for example [11]). This leads then to a noncommutative version of geometric algebra, where the noncommutativity leads to the natural appearance of a spin term [6] and for geometric algebra on the phase space to a natural appearance of supersymmetric quantum mechanics [12].

In the next section we will first give a short overview of the formal structures in the bosonic star product formalism so that one can compare this to the fermionic case. In the third section we will then give a short introduction to geometric algebra in its superanalytic formulation with a Clifford star product. Thereafter, we show how the spin arises naturally from noncommutative geometric algebra and how it can also be formulated in two isomorphic Clifford algebras. Furthermore, we establish the connection of the spin description by spin projectors or spin Wigner functions to the spinor description in [9]. In section six and seven we then describe how the spin can be formulated with a fermionic operator and a fermionic path integral formalism. For both approaches the relation to the fermionic star product formalism is established. In particular we show that the relation of the fermionic path integral and the fermionic star product formalism is analogous to the bosonic one that was found in [13, 14].

\section{The Bosonic Star Product Formalism}

On the $2 d$-dimensional flat phase space with coordinates $\left(q^{i}, p^{i}\right)$ the Moyal product of two phase space functions $f(q, p)$ and $g(q, p)$ is defined as

$$
f *_{M} g=f \exp \left[\frac{\mathrm{i} \hbar}{2} \sum_{i=1}^{d}\left(\frac{\overleftarrow{\partial}}{\partial q^{i}} \frac{\vec{\partial}}{\partial p^{i}}-\frac{\overleftarrow{\partial}}{\partial p^{i}} \frac{\vec{\partial}}{\partial q^{i}}\right)\right] g
$$

It can also be written in integral form, which in a two-dimensional phase space reads [15.

$$
f *_{M} g=\frac{1}{\hbar^{2} \pi^{2}} \int d q^{\prime} d q^{\prime \prime} d p^{\prime} d p^{\prime \prime} f\left(q^{\prime}, p^{\prime}\right) g\left(q^{\prime \prime}, p^{\prime \prime}\right) \exp \left(\frac{2}{\mathrm{i} \hbar}\left(p\left(q^{\prime}-q^{\prime \prime}\right)+p^{\prime}\left(q^{\prime \prime}-q\right)+p^{\prime \prime}\left(q-q^{\prime}\right)\right)\right) .
$$

The star product replaces the conventional product between functions on the phase space and it is so constructed that the star anticommutator corresponds to the Poisson bracket:

$$
\lim _{\hbar \rightarrow 0} \frac{1}{\mathrm{i} \hbar}[f, g]_{*_{M}}=\lim _{\hbar \rightarrow 0} \frac{1}{\mathrm{i} \hbar}\left(f *_{M} g-g *_{M} f\right)=\{f, g\}_{P B} .
$$

This relation is the principle of correspondence. The states of a system with Hamilton function $H(q, p)$ are described by Wigner functions $\pi_{n}^{(M)}(q, p)$. The Wigner functions and the corresponding energy levels $E_{n}$ can be calculated with the help of the star exponential $\operatorname{Exp}_{M}(H t)(q, p)$, which is defined as

$$
\operatorname{Exp}_{M}(H t)=e_{*_{M}}^{-\frac{\mathrm{i} t}{\hbar} H}=\sum_{n=0}^{\infty} \frac{1}{n !}\left(\frac{-\mathrm{i} t}{\hbar}\right)^{n} H^{n *_{M}}=\sum_{n=0}^{\infty} \pi_{n}^{(M)} e^{-\mathrm{i} E_{n} t / \hbar}
$$

where $H^{n *_{M}}=H *_{M} \cdots *_{M} H$ is the $n$-fold star product of $H$. The star exponential fulfills the analogue of the time dependent Schrödinger equation

$$
\mathrm{i} \hbar \frac{d}{d t} \operatorname{Exp}_{M}(H t)=H *_{M} \operatorname{Exp}_{M}(H t)
$$


and describes the time development of a phase space function $f(q, p)$ [2]:

$$
f(t)=\overline{\operatorname{Exp}_{M}(H t)} *_{M} f *_{M} \operatorname{Exp}_{M}(H t)
$$

The connection of the star exponential and the path integral was established in [13, 14]. It was shown that the path integral is the Fourier transform of the star exponential, i.e.

$$
\int \frac{D q D p}{2 \pi \hbar} \exp \left[\frac{\mathrm{i}}{\hbar} \int_{t_{i}}^{t_{f}}(p \dot{q}-H) d t\right]=\int \frac{d p}{2 \pi \hbar} \exp \left[\frac{\mathrm{i}}{\hbar} p\left(q_{f}-q_{i}\right)\right] \operatorname{Exp}_{M}(H t)\left(\left(q_{f}+q_{i}\right) / 2, p\right) .
$$

Putting now (2.4) into (2.5) gives the $*_{M}$-eigenvalue equation

$$
H *_{M} \pi_{n}^{(M)}=E_{n} \pi_{n}^{(M)}
$$

and for $t=0$ these equations lead to the spectral decomposition of the Hamilton function:

$$
H=\sum_{n=0}^{\infty} E_{n} \pi_{n}^{(M)}
$$

Substituting this expression in (2.8) gives

$$
\pi_{n}^{(M)} *_{M} \pi_{m}^{(M)}=\delta_{m n} \pi_{n}^{(M)},
$$

which together with the completeness relation $\sum_{n=0}^{\infty} \pi_{n}^{(M)}=1$, that follows from (2.4) for $t=0$, means that the Wigner functions are projectors.

\section{Geometric Algebra and the Clifford Star Product}

In the supersymmetric formulation of geometric algebra, the basisvectors of a $d$-dimensional vector space are Grassmann variables $\boldsymbol{\sigma}_{i}, i=1, \ldots, d$. A general multivector is then a Grassmann number

$$
A=A^{0}+A^{i} \boldsymbol{\sigma}_{i}+A^{i_{1} i_{2}} \boldsymbol{\sigma}_{i_{1}} \boldsymbol{\sigma}_{i_{2}}+\cdots+A^{i_{1} \cdots i_{d}} \boldsymbol{\sigma}_{i_{1}} \cdots \boldsymbol{\sigma}_{i_{d}} .
$$

On this vector space one has the fermionic Clifford star product, which can be derived from the pseudo-classic formalism for fermions in various ways as was shown in [3], [16], or [4]:

$$
A *_{C} B=A \exp \left[\eta_{i j} \frac{\overleftarrow{\partial}}{\partial \boldsymbol{\sigma}_{i}} \frac{\vec{\partial}}{\partial \boldsymbol{\sigma}_{j}}\right] B
$$

where $\eta_{i j}$ is the metric. In three euclidian dimensions for example, the general multivector has a scalar, a vector, a bivector and a pseudoscalar part, i.e. $A=\langle A\rangle_{0}+\langle A\rangle_{1}+\langle A\rangle_{2}+\langle A\rangle_{3}$, where \langle\rangle$_{n}$ projects on the term of Grassmann grade $n$. In the euclidian case the Clifford star product can just as the Moyal product be written in integral form. One has

$$
A *_{C} B=\int d^{3} \boldsymbol{\sigma}^{\prime} d^{3} \boldsymbol{\sigma}^{\prime \prime} A\left(\overrightarrow{\boldsymbol{\sigma}}^{\prime}\right) B\left(\overrightarrow{\boldsymbol{\sigma}}^{\prime \prime}\right) \exp \left[\sum_{i=1}^{3}\left(\boldsymbol{\sigma}_{i} \boldsymbol{\sigma}_{i}^{\prime}+\boldsymbol{\sigma}_{i}^{\prime} \boldsymbol{\sigma}_{i}^{\prime \prime}+\boldsymbol{\sigma}_{i}^{\prime \prime} \boldsymbol{\sigma}_{i}\right)\right],
$$


where the integral $\int d^{3} \boldsymbol{\sigma}=\int d \boldsymbol{\sigma}_{3} \int d \boldsymbol{\sigma}_{2} \int d \boldsymbol{\sigma}_{1}$ is the Berezin integral [3] and $\overrightarrow{\boldsymbol{\sigma}}$ is the tuple $\left(\boldsymbol{\sigma}_{1}, \boldsymbol{\sigma}_{2}, \boldsymbol{\sigma}_{3}\right)$ so that $A\left(\overrightarrow{\boldsymbol{\sigma}}^{\prime}\right)$ and $B\left(\overrightarrow{\boldsymbol{\sigma}}^{\prime \prime}\right)$ are the multivectors $A$ and $B$ with $\boldsymbol{\sigma}_{i}$ substituted by $\boldsymbol{\sigma}_{i}^{\prime}$ and $\boldsymbol{\sigma}_{i}^{\prime \prime}$ respectively. All these sets of Grassmann variables are mutually anticommuting. The Clifford star product of two basis vectors is given by $\boldsymbol{\sigma}_{i} *_{C} \boldsymbol{\sigma}_{j}=\boldsymbol{\sigma}_{i} \boldsymbol{\sigma}_{j}+\delta_{i j}$, so that for two vectors $\boldsymbol{a}=a^{i} \boldsymbol{\sigma}_{i}$ and $\boldsymbol{b}=b^{i} \boldsymbol{\sigma}_{i}$ the scalar and the exterior product can be defined as the symmetric and the antisymmetric part of the Clifford star product:

$$
\frac{1}{2}\{\boldsymbol{a}, \boldsymbol{b}\}_{*_{C}}=\boldsymbol{a} \cdot \boldsymbol{b} \quad \text { and } \quad \frac{1}{2}[\boldsymbol{a}, \boldsymbol{b}]_{*_{C}}=\boldsymbol{a} \boldsymbol{b} \equiv \boldsymbol{a} \wedge \boldsymbol{b},
$$

with the star anticommutator $\{A, B\}_{*_{C}}=A *_{C} B+B *_{C} A$ and the star commutator $[A, B]_{*_{C}}=A *_{C} B-B *_{C} A$. The scalar and the exterior product can be generalized to homogenous multivectors $A_{(m)}$ and $B_{(n)}$ of Grassmann grade $m$ and $n$ as $A_{(m)} \cdot B_{(n)}=\left\langle A_{(m)} *_{C} B_{(n)}\right\rangle_{|m-n|}$ and $A_{(m)} B_{(n)}=\left\langle A_{(m)} *_{C} B_{(n)}\right\rangle_{m+n}$.

In three dimensions there are furthermore three basis bivectors

$$
\mathrm{B}_{i}=I_{(3)} *_{C} \boldsymbol{\sigma}_{i},
$$

where $I_{(3)}=\sigma_{1} \sigma_{2} \sigma_{3}$ is the unit pseudoscalar in three dimensions. For the basis bivectors one has

$$
\frac{1}{2}\left\{\mathrm{~B}_{i}, \mathrm{~B}_{j}\right\}_{*_{C}}=-\delta_{i j} \text { and } \frac{1}{2}\left[\mathrm{~B}_{i}, \mathrm{~B}_{j}\right]_{*_{C}}=-\varepsilon_{i j k} \mathrm{~B}_{k} .
$$

And in the basis $\mathrm{i}=\mathrm{B}_{1}, \mathrm{j}=-\mathrm{B}_{2}$ and $\mathrm{k}=\mathrm{B}_{3}$ the bivectors fulfill

$$
\mathrm{i}^{2 *_{C}}=\mathrm{j}^{2 *_{C}}=\mathrm{k}^{2 *_{C}}=\mathrm{i} *_{C} \mathrm{j} *_{C} \mathrm{k}=-1,
$$

so that the even multivector $Q=Q^{0}+Q^{1} \mathrm{i}+Q^{2} \mathrm{j}+Q^{3} \mathrm{k}$ is a quaternion.

The bivectors generate the rotations [17, i.e. a rotation of the vector $\boldsymbol{x}$ around the axis that is given by the unit vector $\boldsymbol{n}$ is generated by the bivector $\mathrm{B}=I_{(3)} *_{C} \boldsymbol{n}$ and the rotated vector $\boldsymbol{x}^{\prime}$ is given by

$$
\boldsymbol{x}^{\prime}=R *_{C} \boldsymbol{x} *_{C} \bar{R},
$$

where $R$ is the rotor, i.e. the fermionic star exponential

$$
R=e_{*_{C}}^{\mathrm{B} \varphi / 2},
$$

and the involution reverses the order of the Grassmann variables, so that $\overline{\mathrm{B}}=-\mathrm{B}$ and, in addition, the scalar coefficients are complex conjugated. A multivector is said to be real if $\bar{A}=A$.

While a vector transforms with a left and a right action of the rotor, a spinor $\psi$ transforms with a left action, i.e.

$$
\psi^{\prime}=R *_{C} \psi
$$

In three dimensions such a spinor can be represented as an even multivector [18]

$$
\psi=\psi^{0}+\psi^{i} \mathrm{~B}_{i}=\left(\psi^{0}+\psi^{3} \mathrm{~B}_{3}\right)+\left(\psi^{2}+\psi^{1} \mathrm{~B}_{1}\right) *_{C} \mathrm{~B}_{2},
$$

which has the tuple analogon

$$
\hat{\psi}=\left(\begin{array}{c}
\psi^{0}+\mathrm{i} \psi^{3} \\
-\psi^{2}+\mathrm{i} \psi^{1}
\end{array}\right)
$$

Specifically for the up and down spinors one has $\psi_{+}=1$ and $\psi_{-}=\mathrm{B}_{2}$. With this choice for the spinor the eigenvalue equation reads

$$
\lambda \psi=\boldsymbol{\sigma}_{i} *_{C} \psi *_{C} \boldsymbol{\sigma}_{3} .
$$

Furthermore $\psi$ as an even multivector can be interpreted as a not-normalized rotor [9]. 


\section{The fermionic star product formalism}

It is now natural to combine the fermionic Clifford product and the bosonic Moyal product to obtain a noncommutative version of geometric algebra. If the coefficients of two vectors do not commute, the square of a vector is in general not a scalar, but one obtains in addition to the scalar a bivector term. For the Hamiltonian of a particle in a homogenous magnetic field with vector potential

$$
\boldsymbol{A}=\frac{1}{2}\left(B_{2} q_{3}-B_{3} q_{2}\right) \boldsymbol{\sigma}_{1}+\frac{1}{2}\left(B_{3} q_{1}-B_{1} q_{3}\right) \boldsymbol{\sigma}_{2}+\frac{1}{2}\left(B_{1} q_{2}-B_{2} q_{1}\right) \boldsymbol{\sigma}_{3}
$$

so that

$$
\boldsymbol{\nabla} \boldsymbol{A}=\left(\frac{\partial}{\partial q_{1}} \boldsymbol{\sigma}_{1}+\frac{\partial}{\partial q_{2}} \boldsymbol{\sigma}_{2}+\frac{\partial}{\partial q_{3}} \boldsymbol{\sigma}_{3}\right) \boldsymbol{A}=\sum_{i=1}^{3} B_{i} \mathrm{~B}_{i}
$$

one gets

$$
\begin{aligned}
H & =\frac{1}{2 m}\left[\left(p_{1}+e A_{1}\right) \boldsymbol{\sigma}_{1}+\left(p_{2}+e A_{2}\right) \boldsymbol{\sigma}_{2}+\left(p_{3}+e A_{3}\right) \boldsymbol{\sigma}_{3}\right]^{2 * M C} \\
& =\frac{1}{2 m} \sum_{i=1}^{3}\left(p_{i}+e A_{i}\right)^{2 *_{M}}+\sum_{i=1}^{3} \epsilon_{i k l} \frac{\hbar \omega_{i}}{4 \mathrm{i}} \boldsymbol{\sigma}_{k} \boldsymbol{\sigma}_{l} \\
& =H_{0}+\mathrm{H}_{S}
\end{aligned}
$$

with $\omega_{i}=\frac{e B_{i}}{m}$. The first term $H_{0}$ describes the Landau problem, which can be solved in the star product formalism as described in 19, 20. The additional bivector valued term $\mathrm{H}_{S}$ describes the spin and one can then in analogy to the Moyal product formalism obtain star exponentials and Wigner functions for the spin. The $*_{C}$-eigenvalue equation reads

$$
\mathrm{H}_{S} *_{C} \pi_{ \pm}^{(C)}=E \pi_{ \pm}^{(C)}
$$

With the assumption that the $*_{C}$-square of the spin term in the Hamiltonian is a positive real value, i.e.

$$
E^{2}=\mathrm{H}_{S} *_{C} \mathrm{H}_{S} \in \mathbb{R}_{+}
$$

which applies not only to all real bivectors but also to all real vectors and some multivectors with vector and bivector part, the $*_{C}$-eigenvalue equation (4.6) is solved by

$$
\pi_{ \pm}^{(C)}=\frac{1}{2}\left(1 \pm \frac{\mathrm{H}_{S}}{|E|}\right)
$$

with $*_{C}$-eigenvalues $E_{ \pm}= \pm|E|$. It is easy to show that these solutions fulfill the projector properties $\pi_{ \pm}^{(C)} *_{C} \pi_{ \pm}^{(C)}=\pi_{ \pm}^{(C)}, \pi_{+}^{(C)} *_{C} \pi_{-}^{(C)}=\pi_{-}^{(C)} *_{C} \pi_{+}^{(C)}=0$, and $\pi_{+}^{(C)}+\pi_{-}^{(C)}=1$. As in the bosonic star product formalism, the star-exponential describes the time development and is defined similarly:

$$
\operatorname{Exp}_{C}\left(\mathrm{H}_{S} t\right)=e_{*_{C}}^{-\mathrm{iH}_{S} t}=\cos (|E| t)-\mathrm{i} \frac{\mathrm{H}_{S}}{|E|} \sin (|E| t) .
$$

It can also be expressed by the Fourier-Dirichlet expansion:

$$
\operatorname{Exp}_{C}\left(\mathrm{H}_{S} t\right)=\sum_{E} \pi_{E}^{(C)} e^{-\mathrm{i} E t}
$$


For the specific example of a magnetic field in $z$-direction with vector potential $\boldsymbol{A}=-\frac{B}{2} q_{2} \boldsymbol{\sigma}_{1}+\frac{B}{2} q_{1} \boldsymbol{\sigma}_{2}$ and Hamiltonian $\mathrm{H}_{S_{z}}=\frac{\hbar \omega}{2 \mathrm{i}} \sigma_{1} \sigma_{2}$ this results in

$$
\begin{aligned}
\pi_{ \pm}^{(C)} & =\frac{1}{2}\left(1 \mp \mathrm{i} \boldsymbol{\sigma}_{1} \boldsymbol{\sigma}_{2}\right) \\
\text { and } \quad \operatorname{Exp}_{C}\left(\mathrm{H}_{S_{z}} t\right) & =\cos \left(\frac{\hbar \omega}{2} t\right)-\boldsymbol{\sigma}_{1} \boldsymbol{\sigma}_{2} \sin \left(\frac{\hbar \omega}{2} t\right) .
\end{aligned}
$$

With the star exponential it is possible to describe the time development of the generators $\boldsymbol{\sigma}_{i}$ as

$$
\begin{aligned}
& \boldsymbol{\sigma}_{1}(t)=\overline{\operatorname{Exp}_{C}\left(\mathrm{H}_{S_{z}} t\right)} *_{C} \boldsymbol{\sigma}_{1} *_{C} \operatorname{Exp}_{C}\left(\mathrm{H}_{S_{z}} t\right)=\boldsymbol{\sigma}_{1} \cos (\hbar \omega t)-\boldsymbol{\sigma}_{2} \sin (\hbar \omega t), \\
& \boldsymbol{\sigma}_{2}(t)=\overline{\operatorname{Exp}_{C}\left(\mathrm{H}_{S_{z}} t\right)} *_{C} \boldsymbol{\sigma}_{2} *_{C} \operatorname{Exp}_{C}\left(\mathrm{H}_{S_{z}} t\right)=\boldsymbol{\sigma}_{1} \sin (\hbar \omega t)+\boldsymbol{\sigma}_{2} \cos (\hbar \omega t), \\
& \boldsymbol{\sigma}_{3}(t)=\overline{\operatorname{Exp}_{C}\left(\mathrm{H}_{S_{z}} t\right)} *_{C} \boldsymbol{\sigma}_{3} *_{C} \operatorname{Exp}_{C}\left(\mathrm{H}_{S_{z}} t\right)=\boldsymbol{\sigma}_{3} .
\end{aligned}
$$

The lowering and raising operator for this case can be derived easily with $\sigma_{1} *_{C} \pi_{ \pm}^{(C)} *_{C} \sigma_{1}=\pi_{\mp}^{(C)}$. Using the projector properties of the Wigner function and defining $\boldsymbol{f}=\pi_{+}^{(C)} *_{C} \boldsymbol{\sigma}_{1}=\frac{1}{2}\left(\boldsymbol{\sigma}_{1}+\mathrm{i} \boldsymbol{\sigma}_{2}\right)$ and $\overline{\boldsymbol{f}}=\boldsymbol{\sigma}_{1} *_{C} \pi_{+}^{(C)}=$ $\frac{1}{2}\left(\sigma_{1}-\mathrm{i} \boldsymbol{\sigma}_{2}\right)$ this results in

$$
\begin{aligned}
\bar{f} *_{C} \pi_{+}^{(C)} *_{C} \boldsymbol{f} & =\pi_{-}^{(C)} \\
\text { and } \quad \boldsymbol{f} *_{C} \pi_{-}^{(C)} *_{C} \overline{\boldsymbol{f}} & =\pi_{+}^{(C)} .
\end{aligned}
$$

Alternatively $\boldsymbol{f}^{\prime}=\pi_{+}^{(C)} *_{C} \boldsymbol{\sigma}_{2}=\frac{1}{2}\left(\boldsymbol{\sigma}_{2}-\mathrm{i} \boldsymbol{\sigma}_{1}\right)$ and $\overline{\boldsymbol{f}}^{\prime}=\boldsymbol{\sigma}_{2} *_{C} \pi_{+}^{(C)}=\frac{1}{2}\left(\boldsymbol{\sigma}_{2}+\mathrm{i} \boldsymbol{\sigma}_{1}\right)$ could be chosen.

Due to the fact that the spin is described by bivector-valued terms, the ladder operators should also be elements of the even subalgebra $\mathcal{C} \ell_{3}^{+}(\mathbb{C})$. The obvious choice is to multiply with $\boldsymbol{\sigma}_{3}$ as it commutes with the Wigner functions and one gets

$$
\begin{aligned}
\mathrm{f} & =\boldsymbol{\sigma}_{3} *_{C} \boldsymbol{f}=\frac{1}{2}\left(\boldsymbol{\sigma}_{3} \boldsymbol{\sigma}_{1}-\mathrm{i} \boldsymbol{\sigma}_{2} \boldsymbol{\sigma}_{3}\right) \\
\text { and } \quad \overline{\mathrm{f}} & =\overline{\boldsymbol{f}} *_{C} \boldsymbol{\sigma}_{3}=-\frac{1}{2}\left(\boldsymbol{\sigma}_{3} \boldsymbol{\sigma}_{1}+\mathrm{i} \boldsymbol{\sigma}_{2} \boldsymbol{\sigma}_{3}\right)
\end{aligned}
$$

or, alternatively, $\mathrm{f}^{\prime}=-\frac{1}{2}\left(\boldsymbol{\sigma}_{2} \boldsymbol{\sigma}_{3}+\mathrm{i} \sigma_{3} \sigma_{1}\right)$ and $\overline{\mathrm{f}}^{\prime}=\frac{1}{2}\left(\boldsymbol{\sigma}_{2} \boldsymbol{\sigma}_{3}-\mathrm{i} \sigma_{3} \sigma_{1}\right)$ respectively so that

$$
\begin{aligned}
\overline{\mathrm{f}} *_{C} \pi_{+}^{(C)} *_{C} \mathrm{f} & =\pi_{-}^{(C)} \\
\text { and } \quad \mathrm{f} *_{C} \pi_{-}^{(C)} *_{C} \overline{\mathrm{f}} & =\pi_{+}^{(C)} .
\end{aligned}
$$

\section{$5 \quad$ Isomorphic Clifford algebras}

There are two isomorphic Clifford algebras to $\mathcal{C} \ell_{3}^{+}(\mathbb{C})$. The first algebra is the complex algebra with only two Grassmann variables $\mathcal{C} \ell_{2}(\mathbb{C})$. One gets the isomorphism by substituting $-\mathrm{i} \boldsymbol{\sigma}_{2} \boldsymbol{\sigma}_{3}$ with $\boldsymbol{\sigma}_{1}$ and $-\mathrm{i} \boldsymbol{\sigma}_{3} \boldsymbol{\sigma}_{1}$ with $\boldsymbol{\sigma}_{2}$. Then the spin can be described with the elements of $\mathcal{C} \ell_{2}(\mathbb{C})$ in almost the same manner. The Hamiltonian, the Wigner-function, the star exponential, and the ladder operators for the spin in $z$-direction are still the same whereas the Hamiltonian for the spin in $x$ - and $y$-direction is $\boldsymbol{H}_{S_{x}}=\frac{\hbar \omega}{2} \boldsymbol{\sigma}_{1}$ and $\boldsymbol{H}_{S_{y}}=\frac{\hbar \omega}{2} \boldsymbol{\sigma}_{2}$ 
respectively so that there are with regard to the star product three anticommuting variables $\sigma_{1}, \sigma_{2}$, and $-\mathrm{i} \boldsymbol{\sigma}_{1} \boldsymbol{\sigma}_{2}$. These can also be formulated in terms of the ladder operators $\boldsymbol{f}$ and $\overline{\boldsymbol{f}}$ from the previous chapter as

$$
\begin{aligned}
\boldsymbol{\sigma}_{1} & =\boldsymbol{f}+\overline{\boldsymbol{f}} \\
\boldsymbol{\sigma}_{2} & =-\mathrm{i}(\boldsymbol{f}-\overline{\boldsymbol{f}}) \\
-\mathrm{i} \sigma_{1} \sigma_{2} & =2 \boldsymbol{f} \overline{\boldsymbol{f}}=2\left(\boldsymbol{f} *_{C} \overline{\boldsymbol{f}}-1\right)
\end{aligned}
$$

and thus the Wigner functions for the spin in $z$-direction can be written as

$$
\begin{aligned}
\pi_{+}^{(C)} & =\boldsymbol{f} *_{C} \overline{\boldsymbol{f}} \\
\text { and } \quad \pi_{-}^{(C)} & =\overline{\boldsymbol{f}} *_{C} \boldsymbol{f} .
\end{aligned}
$$

Therefore, these ladder operators correspond to the holomorphic variables in the bosonic case. This description was for example used in [21. Since this is a linear transformation in $\boldsymbol{\sigma}_{1}$ and $\boldsymbol{\sigma}_{2}$, integral formulations can be translated by using the substitution rule for Grassmann variables [9]:

$$
\int d \boldsymbol{\sigma}_{2} \boldsymbol{\sigma}_{1}=\operatorname{det}\left|\frac{\partial \overrightarrow{\boldsymbol{\sigma}}^{\prime}}{\partial \overrightarrow{\boldsymbol{\sigma}}}\right| \int d \boldsymbol{\sigma}_{2}^{\prime} \boldsymbol{\sigma}_{1}^{\prime}
$$

Additionally, these are just the operators that are used as creation and annihilation operators in coherent state formalisms (see for example [5]).

Up to now the spin was described using complex geometric algebras, but the spinors as described in 9 ] are even elements of the real geometric algebra $\mathcal{C} \ell_{3}(\mathbb{R})$. However, this is the second algebra that is isomorphic to $\mathcal{C} \ell_{3}^{+}(\mathbb{C})$ by simply substituting the imaginary unit i with the pseudoscalar $I_{(3)}$ (and consequently $-\mathrm{i} \sigma_{1} \sigma_{2}$ with $\sigma_{3}$ and so forth). In this algebra the spin term of the Hamiltonian for a homogenous magnetic field in $z$-direction is then a vector $\boldsymbol{H}_{S_{z}}=\frac{\hbar \omega}{2} \boldsymbol{\sigma}_{3}$, but as the assumption for the $*_{C}$-eigenvalue equation (4.7) still holds, the solution is

$$
\pi_{ \pm}^{(C)}=\frac{1}{2}\left(1 \pm \frac{\boldsymbol{H}_{S_{z}}}{|E|}\right)=\frac{1}{2}\left(1 \pm \boldsymbol{\sigma}_{3}\right)
$$

The definition of the star exponential changes accordingly:

$$
\begin{aligned}
\operatorname{Exp}_{C}\left(\boldsymbol{H}_{S_{z}} t\right) & =e_{*_{C}}^{-I_{(3)} *_{C} \boldsymbol{H}_{S_{z}} t}=\cos (|E| t)-I_{(3)} *_{C} \frac{\boldsymbol{H}_{S_{z}}}{|E|} \sin (|E| t) \\
& =\sum_{E} \pi_{E}^{(C)} *_{C} e_{*_{C}}^{-I_{(3)} E t}
\end{aligned}
$$

Now there is a direct connection between the Wigner function $\pi^{(C)}$ for an arbitrary spin direction and the spinor $\psi$. If $\psi$ is normalized, i.e.

$$
\bar{\psi} *_{C} \psi=\psi *_{C} \bar{\psi}=1
$$

the spinor is simply the rotor which rotates the spin-up Wigner function so that $\pi^{(C)}$ can be defined as

$$
\pi^{(C)}=\psi *_{C} \frac{1}{2}\left(1+\sigma_{3}\right) *_{C} \bar{\psi}
$$


With this definition the general properties of the Wigner function still have to be met. First, the idempotence of the Wigner-function is shown:

$$
\begin{aligned}
\pi^{(C)} *_{C} \pi^{(C)} & =\psi *_{C} \frac{1}{2}\left(1+\sigma_{3}\right) *_{C} \bar{\psi} *_{C} \psi *_{C} \frac{1}{2}\left(1+\sigma_{3}\right) *_{C} \bar{\psi} \\
& =\psi *_{C} \frac{1}{4}\left(1+\sigma_{3}\right) *_{C}\left(1+\sigma_{3}\right) *_{C} \bar{\psi} \\
& =\psi *_{C} \frac{1}{2}\left(1+\sigma_{3}\right) *_{C} \bar{\psi} \\
& =\pi^{(C)}
\end{aligned}
$$

To prove the equivalence of the eigenvalue equations in the two formalisms, it is necessary to verify the implication in both directions. With simple algebraic transformations the first direction yields

$$
\begin{array}{ll} 
& \lambda \psi=\boldsymbol{\sigma}_{i} *_{C} \psi *_{C} \boldsymbol{\sigma}_{3} \\
\Rightarrow \quad & \lambda \psi *_{C} \frac{1}{2}\left(1+\boldsymbol{\sigma}_{3}\right) *_{C} \bar{\psi}=\boldsymbol{\sigma}_{i} *_{C} \psi *_{C} \boldsymbol{\sigma}_{3} *_{C} \frac{1}{2}\left(1+\boldsymbol{\sigma}_{3}\right) *_{C} \bar{\psi} \\
\Rightarrow \quad & \lambda \psi *_{C} \frac{1}{2}\left(1+\boldsymbol{\sigma}_{3}\right) *_{C} \bar{\psi}=\boldsymbol{\sigma}_{i} *_{C} \psi *_{C} \frac{1}{2}\left(1+\boldsymbol{\sigma}_{3}\right) *_{C} \bar{\psi} \\
\Rightarrow & \lambda \pi=\boldsymbol{\sigma}_{i} *_{C} \pi^{(C)}
\end{array}
$$

and the other direction

$$
\begin{array}{ll} 
& \lambda \pi^{(C)}=\boldsymbol{\sigma}_{i} *_{C} \pi^{(C)} \\
\Rightarrow & \lambda \psi *_{C}\left(1+\boldsymbol{\sigma}_{3}\right) *_{C} \bar{\psi}=\boldsymbol{\sigma}_{i} *_{C} \psi *_{C}\left(1+\boldsymbol{\sigma}_{3}\right) *_{C} \bar{\psi} \\
\Rightarrow & \lambda \psi+\lambda \psi *_{C} \boldsymbol{\sigma}_{3}=\boldsymbol{\sigma}_{i} *_{C} \psi+\boldsymbol{\sigma}_{i} *_{C} \psi *_{C} \boldsymbol{\sigma}_{3} \\
\Rightarrow \quad & \lambda \psi+\lambda \psi *_{C} \boldsymbol{\sigma}_{3}-\boldsymbol{\sigma}_{i} *_{C} \psi *_{C} \boldsymbol{\sigma}_{3}-\boldsymbol{\sigma}_{i} *_{C} \psi *_{C} \boldsymbol{\sigma}_{3} *_{C} \boldsymbol{\sigma}_{3}=0 \\
\Rightarrow \quad & \left(\lambda \psi-\boldsymbol{\sigma}_{i} *_{C} \psi *_{C} \boldsymbol{\sigma}_{3}\right) *_{C}\left(1+\boldsymbol{\sigma}_{3}\right)=0 \\
\Rightarrow \quad & \lambda \psi=\boldsymbol{\sigma}_{i} *_{C} \psi *_{C} \boldsymbol{\sigma}_{3} .
\end{array}
$$

Choosing again the spin in $z$-direction and inserting $\psi_{ \pm}$from (3.11) into (5.8) yields the Wigner functions from (5.4) as expected. The raising and lowering operator for these functions resulting from (4.16) and (4.17) using the isomorphism are

$$
\begin{aligned}
f & =\frac{1}{2}\left(\boldsymbol{\sigma}_{1}+I_{(3)} *_{C} \boldsymbol{\sigma}_{2}\right) \\
\text { and } \quad \bar{f} & =\frac{1}{2}\left(\boldsymbol{\sigma}_{1}-I_{(3)} *_{C} \boldsymbol{\sigma}_{2}\right),
\end{aligned}
$$

which is the Wigner function (5.4) multiplied with $\sigma_{1}$ (depending on the direction from which $\sigma_{1}$ is multiplied and whether $\pi_{+}$or $\pi_{-}$is chosen one gets $f$ or $\bar{f}$ ). So these multivectors essentially reflect the Wigner function at the $\boldsymbol{\sigma}_{1}$-axis and project onto either the up- or down-spin:

$$
\begin{aligned}
& \bar{f} *_{C} \pi_{+}^{(C)} *_{C} f=\pi_{-}^{(C)} *_{C} \boldsymbol{\sigma}_{1} *_{C} \pi_{+}^{(C)} *_{C} \boldsymbol{\sigma}_{1} *_{C} \pi_{-}^{(C)}=\pi_{-}^{(C)} \\
& f *_{C} \pi_{-}^{(C)} *_{C} \bar{f}=\pi_{+}^{(C)} *_{C} \boldsymbol{\sigma}_{1} *_{C} \pi_{-}^{(C)} *_{C} \boldsymbol{\sigma}_{1} *_{C} \pi_{+}^{(C)}=\pi_{+}^{(C)} .
\end{aligned}
$$




\section{Fermionic operator formalism}

Going back to the complex algebra with three Grassmann variables, the Clifford star product can also be expressed by an operator product by substituting $\boldsymbol{\sigma}_{i}$ with $\hat{\boldsymbol{\sigma}}_{i}=\left(\boldsymbol{\sigma}_{i}+\frac{\vec{\partial}}{\partial \boldsymbol{\sigma}_{i}}\right)$ on the left side of the product. This results in the same Hamiltonian as in 22 .

$$
\hat{\mathrm{H}}_{S}=-|E| \mathrm{i}\left(\boldsymbol{\sigma}_{1}+\frac{\vec{\partial}}{\partial \boldsymbol{\sigma}_{1}}\right)\left(\boldsymbol{\sigma}_{2}+\frac{\vec{\partial}}{\partial \boldsymbol{\sigma}_{2}}\right)
$$

and shows that the fermionic wave function and the Wigner function are proportional because $\hat{\mathrm{H}}_{S} \psi=\mathrm{H}_{S} *_{C} \psi$. The normalization is determined by a newly defined scalar-product

$$
\int d^{3} \boldsymbol{\sigma} \tilde{\psi}(\overrightarrow{\boldsymbol{\sigma}}) \psi(\overrightarrow{\boldsymbol{\sigma}})=\int d \boldsymbol{\sigma}_{3} d \boldsymbol{\sigma}_{2} d \boldsymbol{\sigma}_{1} \tilde{\psi}(\overrightarrow{\boldsymbol{\sigma}}) \psi(\overrightarrow{\boldsymbol{\sigma}})=1,
$$

where $\tilde{\psi}(\overrightarrow{\boldsymbol{\sigma}})$ is defined as

$$
\tilde{\psi}(\overrightarrow{\boldsymbol{\sigma}})=\hat{I}_{(3)} \bar{\psi}(\overrightarrow{\boldsymbol{\sigma}})=I_{(3)} *_{C} \bar{\psi}(\overrightarrow{\boldsymbol{\sigma}})
$$

so that

$$
\int d^{3} \boldsymbol{\sigma} \tilde{\psi}(\overrightarrow{\boldsymbol{\sigma}}) \psi(\overrightarrow{\boldsymbol{\sigma}})=\left(\psi^{0}\right)^{2}+\left(\psi^{1}\right)^{2}+\left(\psi^{2}\right)^{2}+\left(\psi^{3}\right)^{2}+\left(\psi^{12}\right)^{2}+\left(\psi^{23}\right)^{2}+\left(\psi^{31}\right)^{2}+\left(\psi^{123}\right)^{2} .
$$

This yields the following fermionic wave function:

$$
\psi_{ \pm}(\overrightarrow{\boldsymbol{\sigma}})=\sqrt{2} \pi_{ \pm}^{(C)}=\frac{1}{\sqrt{2}}\left(1 \mp \mathrm{i} \boldsymbol{\sigma}_{1} \boldsymbol{\sigma}_{2}\right) .
$$

In Dirac notation this can be written as

$$
\begin{aligned}
\langle\overrightarrow{\boldsymbol{\sigma}} \mid \psi\rangle & =\psi(\overrightarrow{\boldsymbol{\sigma}}) \\
\text { and } \quad\langle\psi \mid \overrightarrow{\boldsymbol{\sigma}}\rangle & =\tilde{\psi}(\overrightarrow{\boldsymbol{\sigma}})
\end{aligned}
$$

so that

$$
\left\langle\psi^{\prime} \mid \psi\right\rangle=\int d^{3} \boldsymbol{\sigma} \tilde{\psi}^{\prime}(\overrightarrow{\boldsymbol{\sigma}}) \psi(\overrightarrow{\boldsymbol{\sigma}})=\int d^{3} \boldsymbol{\sigma}\left\langle\psi^{\prime} \mid \overrightarrow{\boldsymbol{\sigma}}\right\rangle\langle\overrightarrow{\boldsymbol{\sigma}} \mid \psi\rangle
$$

which means that

$$
\int d^{3} \boldsymbol{\sigma}|\overrightarrow{\boldsymbol{\sigma}}\rangle\langle\overrightarrow{\boldsymbol{\sigma}}|=\hat{1}
$$

With the Fourier-transform as defined in [3] but with slightly reordered factors

$$
\begin{aligned}
F\left(\overrightarrow{\boldsymbol{\sigma}}^{\prime}\right) & =\int d^{3} \boldsymbol{\sigma} f(\overrightarrow{\boldsymbol{\sigma}}) e^{\mathrm{i} \boldsymbol{\sigma} \overrightarrow{\boldsymbol{\sigma}}^{\prime}} \\
f(\overrightarrow{\boldsymbol{\sigma}}) & =-\mathrm{i} \int d^{3} \boldsymbol{\sigma}^{\prime} F\left(\overrightarrow{\boldsymbol{\sigma}}^{\prime}\right) e^{\mathrm{i} \overrightarrow{\boldsymbol{\sigma}}^{\prime} \overrightarrow{\boldsymbol{\sigma}}},
\end{aligned}
$$

where $\vec{\sigma} \vec{\sigma}^{\prime}=\sum_{i=1}^{3} \sigma_{i} \sigma_{i}^{\prime}$, and the analogous definition of the delta-function

$$
f\left(\overrightarrow{\boldsymbol{\sigma}}^{\prime}\right)=\int d^{3} \boldsymbol{\sigma} \delta^{3}\left(\overrightarrow{\boldsymbol{\sigma}}^{\prime}-\overrightarrow{\boldsymbol{\sigma}}\right) f(\overrightarrow{\boldsymbol{\sigma}}),
$$


the Fourier-transform of the delta-function is $e^{\mathrm{i} \vec{\sigma}^{\prime} \vec{\sigma}}$. The delta function itself can be derived easily:

$$
\begin{aligned}
\delta^{3}\left(\overrightarrow{\boldsymbol{\sigma}}^{\prime}-\overrightarrow{\boldsymbol{\sigma}}\right) & =-\mathrm{i} \int d^{3} \boldsymbol{\sigma}^{\prime \prime} e^{\mathrm{i} \overrightarrow{\boldsymbol{\sigma}}^{\prime \prime}\left(\overrightarrow{\boldsymbol{\sigma}}-\overrightarrow{\boldsymbol{\sigma}}^{\prime}\right)} \\
& =\int d^{3} \boldsymbol{\sigma}^{\prime \prime} e^{\overrightarrow{\boldsymbol{\sigma}}^{\prime \prime}\left(\overrightarrow{\boldsymbol{\sigma}}^{\prime}-\overrightarrow{\boldsymbol{\sigma}}\right)} \\
& =\left(\boldsymbol{\sigma}_{1}-\boldsymbol{\sigma}_{1}^{\prime}\right)\left(\boldsymbol{\sigma}_{2}-\boldsymbol{\sigma}_{2}^{\prime}\right)\left(\boldsymbol{\sigma}_{3}-\boldsymbol{\sigma}_{3}^{\prime}\right) .
\end{aligned}
$$

In addition, it can be noted using (3.3) and (6.13) that within an integral the star product, which is the same as the operator product, is identical to the conventional product, i.e.

$$
\begin{aligned}
\int d^{3} \boldsymbol{\sigma} \hat{A}(\overrightarrow{\boldsymbol{\sigma}}) B(\overrightarrow{\boldsymbol{\sigma}}) & =\int d^{3} \boldsymbol{\sigma} A(\overrightarrow{\boldsymbol{\sigma}}) *_{C} B(\overrightarrow{\boldsymbol{\sigma}}) \\
& =\int d^{3} \boldsymbol{\sigma} d^{3} \boldsymbol{\sigma}^{\prime} d^{3} \boldsymbol{\sigma}^{\prime \prime} A\left(\overrightarrow{\boldsymbol{\sigma}}^{\prime}\right) B\left(\overrightarrow{\boldsymbol{\sigma}}^{\prime \prime}\right) e^{\left(\overrightarrow{\boldsymbol{\sigma}} \overrightarrow{\boldsymbol{\sigma}}^{\prime}+\overrightarrow{\boldsymbol{\sigma}}^{\prime} \overrightarrow{\boldsymbol{\sigma}}^{\prime \prime}+\overrightarrow{\boldsymbol{\sigma}}^{\prime \prime} \overrightarrow{\boldsymbol{\sigma}}\right)} \\
& =\int d^{3} \boldsymbol{\sigma}^{\prime} d^{3} \boldsymbol{\sigma}^{\prime \prime} d^{3} \boldsymbol{\sigma} e^{\overrightarrow{\boldsymbol{\sigma}}\left(\overrightarrow{\boldsymbol{\sigma}}^{\prime}-\overrightarrow{\boldsymbol{\sigma}}^{\prime \prime}\right)} A\left(\overrightarrow{\boldsymbol{\sigma}}^{\prime}\right) B\left(\overrightarrow{\boldsymbol{\sigma}}^{\prime \prime}\right) e^{\overrightarrow{\boldsymbol{\sigma}}^{\prime} \overrightarrow{\boldsymbol{\sigma}}^{\prime \prime}} \\
& =\int d^{3} \boldsymbol{\sigma}^{\prime} d^{3} \boldsymbol{\sigma}^{\prime \prime} \delta^{3}\left(\overrightarrow{\boldsymbol{\sigma}}^{\prime}-\overrightarrow{\boldsymbol{\sigma}}^{\prime \prime}\right) A\left(\overrightarrow{\boldsymbol{\sigma}}^{\prime}\right) B\left(\overrightarrow{\boldsymbol{\sigma}}^{\prime \prime}\right) e^{\overrightarrow{\boldsymbol{\sigma}}^{\prime} \overrightarrow{\boldsymbol{\sigma}}^{\prime \prime}} \\
& =\int d^{3} \boldsymbol{\sigma}^{\prime} A\left(\overrightarrow{\boldsymbol{\sigma}}^{\prime}\right) B\left(\overrightarrow{\boldsymbol{\sigma}}^{\prime}\right) e^{\overrightarrow{\boldsymbol{\sigma}}^{\prime} \overrightarrow{\boldsymbol{\sigma}}^{\prime}} \\
& =\int d^{3} \boldsymbol{\sigma} A(\overrightarrow{\boldsymbol{\sigma}}) B(\overrightarrow{\boldsymbol{\sigma}}),
\end{aligned}
$$

just as in the bosonic case, where $\int d q d p f(q, p) *_{M} g(q, p)=\int d q d p f(q, p) g(q, p)$. With these definitions and results a possible choice for the bras and kets is

$$
\begin{array}{ll}
\langle\psi|=\int d^{3} \boldsymbol{\sigma}^{\prime} \tilde{\psi}\left(\overrightarrow{\boldsymbol{\sigma}}^{\prime}\right) & |\psi\rangle=\psi\left(\overrightarrow{\boldsymbol{\sigma}}^{\prime}\right) \\
\langle\overrightarrow{\boldsymbol{\sigma}}|=\int d^{3} \boldsymbol{\sigma}^{\prime} \delta^{3}\left(\overrightarrow{\boldsymbol{\sigma}}-\overrightarrow{\boldsymbol{\sigma}}^{\prime}\right) & |\overrightarrow{\boldsymbol{\sigma}}\rangle=-\delta^{3}\left(\overrightarrow{\boldsymbol{\sigma}}-\overrightarrow{\boldsymbol{\sigma}}^{\prime}\right),
\end{array}
$$

where the bras are integration operators and all but $|\overrightarrow{\boldsymbol{\sigma}}\rangle$ are even multivectors. This results furthermore in

$$
\begin{aligned}
\left\langle\overrightarrow{\boldsymbol{\sigma}}^{\prime} \mid \overrightarrow{\boldsymbol{\sigma}}\right\rangle & =\delta^{3}\left(\overrightarrow{\boldsymbol{\sigma}}^{\prime}-\overrightarrow{\boldsymbol{\sigma}}\right), \\
\left\langle\overrightarrow{\boldsymbol{\sigma}}^{\prime}\left|\hat{\mathrm{H}}_{S}\right| \overrightarrow{\boldsymbol{\sigma}}\right\rangle & =\hat{\mathrm{H}}_{S}\left(\overrightarrow{\boldsymbol{\sigma}}^{\prime}\right) \delta^{3}\left(\overrightarrow{\boldsymbol{\sigma}}^{\prime}-\overrightarrow{\boldsymbol{\sigma}}\right), \\
\text { and } \quad\left\langle\overrightarrow{\boldsymbol{\sigma}}\left|\hat{\mathrm{H}}_{S}\right| \psi\right\rangle & =\hat{\mathrm{H}}_{S}(\overrightarrow{\boldsymbol{\sigma}}) \psi(\overrightarrow{\boldsymbol{\sigma}}) .
\end{aligned}
$$

The formal solution for the time development of the fermionic wave function can also be expressed with the star-exponential:

$$
\begin{aligned}
\psi(\overrightarrow{\boldsymbol{\sigma}}, t) & =e^{-\mathrm{i}_{S} t} \psi(\overrightarrow{\boldsymbol{\sigma}}) \\
& =\left(\cos (|E| t)-\mathrm{i} \frac{\hat{\mathrm{H}}_{S}}{|E|} \sin (|E| t)\right) \psi(\overrightarrow{\boldsymbol{\sigma}}) \\
& =\operatorname{Exp}_{C}\left(\mathrm{H}_{S} t\right) *_{C} \psi(\overrightarrow{\boldsymbol{\sigma}}) .
\end{aligned}
$$




\section{$7 \quad$ Fermionic path integral}

The fermionic path integral was formulated in $\left[3\right.$, where the time development operator $\hat{G}=e^{-i \hat{H}_{S} t}$ was expressed as a path integral by using the integral form of the operator product. For convenicence, we briefly demonstrate its derivation but in the context of geometric algebra. The indices used here do not depict single generators of a Grassmann algebra but whole sets of generators $\left(\overrightarrow{\boldsymbol{\sigma}}_{t}=\left\{\left(\boldsymbol{\sigma}_{t}\right)_{i}\right\}\right)$. As was shown in the last chapter, the time development operator is simply the star exponential

$$
\hat{G} \psi(\overrightarrow{\boldsymbol{\sigma}})=\operatorname{Exp}_{C}\left(\mathrm{H}_{S} t\right) *_{C} \psi(\overrightarrow{\boldsymbol{\sigma}})=G *_{C} \psi(\overrightarrow{\boldsymbol{\sigma}}) .
$$

This can also be expressed as an infinite product of star exponentials

$$
G=\lim _{N \rightarrow \infty}\left[\operatorname{Exp}_{C}\left(\mathrm{H}_{S} t / N\right)\right]^{N *_{C}}
$$

and using the integral form of the star product (3.3) for the time development (6.26) one gets

$$
G(\overrightarrow{\boldsymbol{\sigma}}, t)=\lim _{N \rightarrow \infty} \int \prod_{m=1}^{N} d^{3} \boldsymbol{\sigma}_{m} d^{3} \boldsymbol{\sigma}_{m}^{\prime} \exp \left(\sum_{n=1}^{N}\left(\overrightarrow{\boldsymbol{\sigma}}_{n} \overrightarrow{\boldsymbol{\sigma}}_{n}^{\prime}+\overrightarrow{\boldsymbol{\sigma}}_{n}^{\prime} \overrightarrow{\boldsymbol{\sigma}}_{n+1}+\overrightarrow{\boldsymbol{\sigma}}_{n+1} \overrightarrow{\boldsymbol{\sigma}}_{n}-\mathrm{iH}_{S}\left(\overrightarrow{\boldsymbol{\sigma}}^{\prime}\right) t / N\right)\right)
$$

where $\overrightarrow{\boldsymbol{\sigma}}_{N+1}=\overrightarrow{\boldsymbol{\sigma}}$. To get a path integral formulation similar to the bosonic case, the integration over $\prod_{m=1}^{N} d^{3} \boldsymbol{\sigma}_{m}$ has to be performed. Therefore, the different sets of variables are separated by substituting $\overrightarrow{\boldsymbol{\sigma}}_{n}$ with $\overrightarrow{\boldsymbol{\sigma}}_{n}^{\prime \prime}+\overrightarrow{\boldsymbol{\sigma}}_{n}^{\prime \prime \prime}$ with new integration variables $\overrightarrow{\boldsymbol{\sigma}}^{\prime \prime}$, which results in the exponent

$$
\begin{aligned}
& \sum_{n=1}^{N-1}\left(\left(\overrightarrow{\boldsymbol{\sigma}}_{n}^{\prime \prime}+\overrightarrow{\boldsymbol{\sigma}}_{n}^{\prime \prime \prime}\right) \overrightarrow{\boldsymbol{\sigma}}_{n}^{\prime}+\overrightarrow{\boldsymbol{\sigma}}_{n}^{\prime}\left(\overrightarrow{\boldsymbol{\sigma}}_{n+1}^{\prime \prime}+\overrightarrow{\boldsymbol{\sigma}}_{n+1}^{\prime \prime \prime}\right)+\left(\overrightarrow{\boldsymbol{\sigma}}_{n+1}^{\prime \prime}+\overrightarrow{\boldsymbol{\sigma}}_{n+1}^{\prime \prime \prime}\right)\left(\overrightarrow{\boldsymbol{\sigma}}_{n}^{\prime \prime}+\overrightarrow{\boldsymbol{\sigma}}_{n}^{\prime \prime \prime}\right)\right) \\
& +\left(\overrightarrow{\boldsymbol{\sigma}}_{N}^{\prime \prime}+\overrightarrow{\boldsymbol{\sigma}}_{N}^{\prime \prime \prime}\right) \overrightarrow{\boldsymbol{\sigma}}_{N}^{\prime}+\overrightarrow{\boldsymbol{\sigma}}_{N}^{\prime} \overrightarrow{\boldsymbol{\sigma}}+\overrightarrow{\boldsymbol{\sigma}}\left(\overrightarrow{\boldsymbol{\sigma}}_{N}^{\prime \prime}+\overrightarrow{\boldsymbol{\sigma}}_{N}^{\prime \prime \prime}\right) \\
= & \sum_{n=1}^{N-1}\left(\overrightarrow{\boldsymbol{\sigma}}_{n}^{\prime}\left(\overrightarrow{\boldsymbol{\sigma}}_{n+1}^{\prime \prime \prime}-\overrightarrow{\boldsymbol{\sigma}}_{n}^{\prime \prime \prime}\right)+\overrightarrow{\boldsymbol{\sigma}}_{n+1}^{\prime \prime \prime} \overrightarrow{\boldsymbol{\sigma}}_{n}^{\prime \prime \prime}\right)+\overrightarrow{\boldsymbol{\sigma}}_{N}^{\prime}\left(\overrightarrow{\boldsymbol{\sigma}}-\overrightarrow{\boldsymbol{\sigma}}_{N}^{\prime \prime \prime}\right)+\overrightarrow{\boldsymbol{\sigma}} \overrightarrow{\boldsymbol{\sigma}}_{N}^{\prime \prime \prime} \\
& +\sum_{n=2}^{N-1}\left(\overrightarrow{\boldsymbol{\sigma}}_{n}^{\prime \prime}\left(\overrightarrow{\boldsymbol{\sigma}}_{n}^{\prime}-\overrightarrow{\boldsymbol{\sigma}}_{n-1}^{\prime}-\overrightarrow{\boldsymbol{\sigma}}_{n+1}^{\prime \prime \prime}+\overrightarrow{\boldsymbol{\sigma}}_{n-1}^{\prime \prime \prime}\right)\right)+\overrightarrow{\boldsymbol{\sigma}}_{1}^{\prime \prime}\left(\overrightarrow{\boldsymbol{\sigma}}_{1}^{\prime}-\overrightarrow{\boldsymbol{\sigma}}_{2}^{\prime \prime \prime}\right)+\overrightarrow{\boldsymbol{\sigma}}_{N}^{\prime \prime}\left(\overrightarrow{\boldsymbol{\sigma}}_{N-1}^{\prime \prime \prime}-\overrightarrow{\boldsymbol{\sigma}}_{N-1}^{\prime}+\overrightarrow{\boldsymbol{\sigma}}_{N}^{\prime}-\overrightarrow{\boldsymbol{\sigma}}\right) \\
& +\sum_{n=1}^{N-1} \overrightarrow{\boldsymbol{\sigma}}_{n+1}^{\prime \prime} \overrightarrow{\boldsymbol{\sigma}}_{n}^{\prime \prime}
\end{aligned}
$$

where the summand with the Hamiltonian is omitted because it is not altered. The mixed terms (7.7) are eliminated by choosing $\overrightarrow{\boldsymbol{\sigma}}_{n+1}^{\prime \prime \prime}-\overrightarrow{\boldsymbol{\sigma}}_{n-1}^{\prime \prime \prime}=\overrightarrow{\boldsymbol{\sigma}}_{n}^{\prime}-\overrightarrow{\boldsymbol{\sigma}}_{n-1}^{\prime}$ for $n=2 \ldots N-1, \overrightarrow{\boldsymbol{\sigma}}_{2}^{\prime \prime \prime}=\overrightarrow{\boldsymbol{\sigma}}_{1}^{\prime}$, and $\overrightarrow{\boldsymbol{\sigma}}_{N-1}^{\prime \prime \prime}=\overrightarrow{\boldsymbol{\sigma}}+\overrightarrow{\boldsymbol{\sigma}}_{N-1}^{\prime}-\overrightarrow{\boldsymbol{\sigma}}_{N}^{\prime}$. Thus, the rest of the $\overrightarrow{\boldsymbol{\sigma}}^{\prime \prime}$-terms (7.8) form the Grassmannian Gauss integral and for even $N$ it yields

$$
\int \prod_{m=1}^{N} d^{3} \overrightarrow{\boldsymbol{\sigma}}_{m}^{\prime \prime} \exp \left(\sum_{n=1}^{N-1} \overrightarrow{\boldsymbol{\sigma}}_{n+1}^{\prime \prime} \overrightarrow{\boldsymbol{\sigma}}_{n}^{\prime \prime}\right)=1
$$


Collecting the remaining terms we get

$$
\begin{aligned}
& \sum_{n=1}^{N-1}\left(\overrightarrow{\boldsymbol{\sigma}}_{n}^{\prime}\left(\overrightarrow{\boldsymbol{\sigma}}_{n+1}^{\prime \prime \prime}-\overrightarrow{\boldsymbol{\sigma}}_{n}^{\prime \prime \prime}\right)+\overrightarrow{\boldsymbol{\sigma}}_{n+1}^{\prime \prime \prime} \overrightarrow{\boldsymbol{\sigma}}_{n}^{\prime \prime \prime}\right)+\overrightarrow{\boldsymbol{\sigma}}_{N}^{\prime}\left(\overrightarrow{\boldsymbol{\sigma}}-\overrightarrow{\boldsymbol{\sigma}}_{N}^{\prime \prime \prime}\right)+\overrightarrow{\boldsymbol{\sigma}} \overrightarrow{\boldsymbol{\sigma}}_{N}^{\prime \prime \prime} \\
= & \sum_{n=1}^{N / 2-1}\left(\overrightarrow{\boldsymbol{\sigma}}_{2 n}^{\prime}\left(\overrightarrow{\boldsymbol{\sigma}}_{2 n+1}^{\prime \prime \prime}-\overrightarrow{\boldsymbol{\sigma}}_{2 n}^{\prime \prime \prime}\right)+\overrightarrow{\boldsymbol{\sigma}}_{2 n+1}^{\prime \prime \prime} \overrightarrow{\boldsymbol{\sigma}}_{2 n}^{\prime \prime \prime}+\overrightarrow{\boldsymbol{\sigma}}_{2 n+1}^{\prime}\left(\overrightarrow{\boldsymbol{\sigma}}_{2 n+2}^{\prime \prime \prime}-\overrightarrow{\boldsymbol{\sigma}}_{2 n+1}^{\prime \prime \prime}\right)+\overrightarrow{\boldsymbol{\sigma}}_{2 n+2}^{\prime \prime \prime} \overrightarrow{\boldsymbol{\sigma}}_{2 n+1}^{\prime \prime \prime}\right) \\
& +\overrightarrow{\boldsymbol{\sigma}}_{1}^{\prime}\left(\overrightarrow{\boldsymbol{\sigma}}_{2}^{\prime \prime \prime}-\overrightarrow{\boldsymbol{\sigma}}_{1}^{\prime \prime \prime}\right)+\overrightarrow{\boldsymbol{\sigma}}_{2}^{\prime \prime \prime} \overrightarrow{\boldsymbol{\sigma}}_{1}^{\prime \prime \prime}+\overrightarrow{\boldsymbol{\sigma}}_{N}^{\prime}\left(\overrightarrow{\boldsymbol{\sigma}}-\overrightarrow{\boldsymbol{\sigma}}_{N}^{\prime \prime \prime}\right)+\overrightarrow{\boldsymbol{\sigma}} \overrightarrow{\boldsymbol{\sigma}}_{N}^{\prime \prime \prime} \\
= & \sum_{n=1}^{N / 2-1}\left(-\overrightarrow{\boldsymbol{\sigma}}_{2 n}^{\prime} \overrightarrow{\boldsymbol{\sigma}}_{2 n}^{\prime \prime \prime}+\overrightarrow{\boldsymbol{\sigma}}_{2 n+1}^{\prime} \overrightarrow{\boldsymbol{\sigma}}_{2 n+2}^{\prime \prime \prime}+\overrightarrow{\boldsymbol{\sigma}}_{2 n+1}^{\prime \prime \prime}\left(\overrightarrow{\boldsymbol{\sigma}}_{2 n+1}^{\prime \prime}-\overrightarrow{\boldsymbol{\sigma}}_{2 n}^{\prime}\right)+\overrightarrow{\boldsymbol{\sigma}}_{2 n+1}^{\prime \prime \prime}\left(\overrightarrow{\boldsymbol{\sigma}}_{2 n}^{\prime \prime \prime}-\overrightarrow{\boldsymbol{\sigma}}_{2 n+2}^{\prime \prime \prime}\right)\right) \\
& +\overrightarrow{\boldsymbol{\sigma}}_{N}^{\prime}\left(\overrightarrow{\boldsymbol{\sigma}}-\overrightarrow{\boldsymbol{\sigma}}_{N}^{\prime \prime \prime}\right)+\overrightarrow{\boldsymbol{\sigma}} \overrightarrow{\boldsymbol{\sigma}}_{N}^{\prime \prime \prime} \\
& \sum_{n=1}^{N / 2} \overrightarrow{\boldsymbol{\sigma}}_{2 n}^{\prime \prime \prime}\left(\overrightarrow{\boldsymbol{\sigma}}_{2 n}^{\prime}-\overrightarrow{\boldsymbol{\sigma}}_{2 n-1}^{\prime}\right)+\overrightarrow{\boldsymbol{\sigma}}\left(\overrightarrow{\boldsymbol{\sigma}}_{N}^{\prime \prime \prime}-\overrightarrow{\boldsymbol{\sigma}}_{N}^{\prime}\right) .
\end{aligned}
$$

In the continual limit the substitution results in $\overrightarrow{\boldsymbol{\sigma}}^{\prime \prime \prime}(\tau)=\frac{1}{2}\left(\overrightarrow{\boldsymbol{\sigma}}^{\prime}(\tau)+\overrightarrow{\boldsymbol{\sigma}}^{\prime}(0)\right)$ so that the complete integral can then formally be written as

$$
G(\overrightarrow{\boldsymbol{\sigma}}, t)=\lim _{N \rightarrow \infty} \int \prod_{n=1}^{N} d^{3} \boldsymbol{\sigma}_{n}^{\prime} \exp \left(\int_{0}^{t}\left[\frac{1}{4} \overrightarrow{\boldsymbol{\sigma}}^{\prime} \dot{\overrightarrow{\boldsymbol{\sigma}}}^{\prime}-\mathrm{iH}_{S}\left(\overrightarrow{\boldsymbol{\sigma}}^{\prime}\right)\right] d \tau+\frac{1}{2}\left(\overrightarrow{\boldsymbol{\sigma}} \overrightarrow{\boldsymbol{\sigma}}_{0}^{\prime}+\overrightarrow{\boldsymbol{\sigma}}_{0}^{\prime} \overrightarrow{\boldsymbol{\sigma}}_{t}^{\prime}+\overrightarrow{\boldsymbol{\sigma}}_{t}^{\prime} \overrightarrow{\boldsymbol{\sigma}}\right)\right)
$$

where $\overrightarrow{\boldsymbol{\sigma}}_{0}^{\prime}=\overrightarrow{\boldsymbol{\sigma}}^{\prime}(0)$ and $\overrightarrow{\boldsymbol{\sigma}}_{t}^{\prime}=\overrightarrow{\boldsymbol{\sigma}}^{\prime}(t)$.

The Dirac notation, which was established in the previous chapter, can be used for an alternative definition of the fermionic Green's function. Using (6.6) and (6.8) with the formal solution for the time development yields

$$
\begin{aligned}
\psi\left(\overrightarrow{\boldsymbol{\sigma}}_{t}, t\right) & =\left\langle\overrightarrow{\boldsymbol{\sigma}}_{t} \mid \psi(t)\right\rangle \\
& =\left\langle\overrightarrow{\boldsymbol{\sigma}}_{t}\left|e^{-\mathrm{i}_{S}\left(t-t_{0}\right)}\right| \psi\left(t_{0}\right)\right\rangle \\
& =\left\langle\overrightarrow{\boldsymbol{\sigma}}_{t}\left|e^{-\mathrm{i} \hat{\mathrm{H}}_{S}\left(t-t_{0}\right)} \int d^{3} \boldsymbol{\sigma}_{t_{0}}\right| \overrightarrow{\boldsymbol{\sigma}}_{t_{0}}\right\rangle\left\langle\overrightarrow{\boldsymbol{\sigma}}_{t_{0}} \mid \psi\left(t_{0}\right)\right\rangle \\
& =\int d^{3} \boldsymbol{\sigma}_{t_{0}}\left\langle\overrightarrow{\boldsymbol{\sigma}}_{t}\left|e^{-\mathrm{i} \hat{\mathrm{H}}_{S}\left(t-t_{0}\right)}\right| \overrightarrow{\boldsymbol{\sigma}}_{t_{0}}\right\rangle \psi\left(\overrightarrow{\boldsymbol{\sigma}}_{t_{0}}, t_{0}\right) \\
& =\int d^{3} \boldsymbol{\sigma}_{t_{0}} G\left(\overrightarrow{\boldsymbol{\sigma}}_{t}, t ; \overrightarrow{\boldsymbol{\sigma}}_{t_{0}}, t_{0}\right) \psi\left(\overrightarrow{\boldsymbol{\sigma}}_{t_{0}}, t_{0}\right)
\end{aligned}
$$

So the fermionic Green's function is

$$
G\left(\overrightarrow{\boldsymbol{\sigma}}_{t}, t ; \overrightarrow{\boldsymbol{\sigma}}_{t_{0}}, t_{0}\right)=\left\langle\overrightarrow{\boldsymbol{\sigma}}_{t}\left|e^{-\mathrm{i} \hat{\mathrm{H}}_{S}\left(t-t_{0}\right)}\right| \overrightarrow{\boldsymbol{\sigma}}_{t_{0}}\right\rangle
$$


With (6.24), (3.3), and (6.11) this results in

$$
\begin{aligned}
G\left(\overrightarrow{\boldsymbol{\sigma}}_{t}, t ; \overrightarrow{\boldsymbol{\sigma}}_{t_{0}}, t_{0}\right) & =e^{-\mathrm{i} \hat{\mathrm{H}}_{S}\left(\overrightarrow{\boldsymbol{\sigma}}_{t}\right)\left(t-t_{0}\right)} \delta^{3}\left(\overrightarrow{\boldsymbol{\sigma}}_{t}-\overrightarrow{\boldsymbol{\sigma}}_{t_{0}}\right) \\
& =\operatorname{Exp}_{C}\left(\mathrm{H}_{S}\left(\overrightarrow{\boldsymbol{\sigma}}_{t}\right)\left(t-t_{0}\right)\right) *_{C} \delta^{3}\left(\overrightarrow{\boldsymbol{\sigma}}_{t}-\overrightarrow{\boldsymbol{\sigma}}_{t_{0}}\right) \\
& =\int d^{3} \boldsymbol{\sigma}^{\prime} d^{3} \boldsymbol{\sigma}^{\prime \prime} \operatorname{Exp}_{C}\left(\mathrm{H}_{S}\left(\overrightarrow{\boldsymbol{\sigma}}^{\prime}\right)\left(t-t_{0}\right)\right) \delta^{3}\left(\overrightarrow{\boldsymbol{\sigma}}^{\prime \prime}-\overrightarrow{\boldsymbol{\sigma}}_{t_{0}}\right) e^{\overrightarrow{\boldsymbol{\sigma}}_{t} \overrightarrow{\boldsymbol{\sigma}}^{\prime}+\overrightarrow{\boldsymbol{\sigma}}^{\prime} \overrightarrow{\boldsymbol{\sigma}}^{\prime \prime}+\overrightarrow{\boldsymbol{\sigma}}^{\prime \prime} \overrightarrow{\boldsymbol{\sigma}}_{t}} \\
& =-\int d^{3} \boldsymbol{\sigma}^{\prime} \operatorname{Exp}_{C}\left(\mathrm{H}_{S}\left(\overrightarrow{\boldsymbol{\sigma}}^{\prime}\right)\left(t-t_{0}\right)\right) e^{\overrightarrow{\boldsymbol{\sigma}}_{t} \overrightarrow{\boldsymbol{\sigma}}^{\prime}+\overrightarrow{\boldsymbol{\sigma}}^{\prime} \overrightarrow{\boldsymbol{\sigma}}_{t_{0}}+\overrightarrow{\boldsymbol{\sigma}}_{t_{0}} \overrightarrow{\boldsymbol{\sigma}}_{t}}
\end{aligned}
$$

Substituting now $\overrightarrow{\boldsymbol{\sigma}}^{\prime}$ with $\overrightarrow{\boldsymbol{\sigma}}_{t}-\overrightarrow{\boldsymbol{\sigma}}^{\prime}$ yields

$$
\begin{aligned}
G\left(\overrightarrow{\boldsymbol{\sigma}}_{t}, t ; \overrightarrow{\boldsymbol{\sigma}}_{t_{0}}, t_{0}\right) & =\int d^{3} \boldsymbol{\sigma}^{\prime} \operatorname{Exp}_{C}\left(\mathrm{H}_{S}\left(\overrightarrow{\boldsymbol{\sigma}}_{t}-\overrightarrow{\boldsymbol{\sigma}}^{\prime}\right)\left(t-t_{0}\right)\right) e^{\overrightarrow{\boldsymbol{\sigma}}^{\prime} \overrightarrow{\boldsymbol{\sigma}}_{t}+\overrightarrow{\boldsymbol{\sigma}}_{t_{0}} \overrightarrow{\boldsymbol{\sigma}}^{\prime}} \\
& =\int d^{3} \boldsymbol{\sigma}^{\prime} \operatorname{Exp}_{C}\left(\mathrm{H}_{S}\left(\overrightarrow{\boldsymbol{\sigma}}_{t}-\overrightarrow{\boldsymbol{\sigma}}^{\prime}\right)\left(t-t_{0}\right)\right) e^{\overrightarrow{\boldsymbol{\sigma}}^{\prime}\left(\overrightarrow{\boldsymbol{\sigma}}_{t}-\overrightarrow{\boldsymbol{\sigma}}_{t_{0}}\right)}
\end{aligned}
$$

So, with the alternative definition of the Green's function one gets a connection to the star exponential that is similar to the connection in the bosonic case 13,14 . The same result can be obtained in another way. $G\left(\overrightarrow{\boldsymbol{\sigma}}_{t}, t ; \overrightarrow{\boldsymbol{\sigma}}_{t_{0}}, t_{0}\right)$ in (7.21) can be subdivided into infinitesimal components

$$
\begin{aligned}
G\left(\overrightarrow{\boldsymbol{\sigma}}_{t}, t ; \overrightarrow{\boldsymbol{\sigma}}_{t_{0}}, t_{0}\right) & =\lim _{N \rightarrow \infty} \int \prod_{m=1}^{N} d^{3} \boldsymbol{\sigma}_{m} \prod_{n=N}^{0} G\left(\overrightarrow{\boldsymbol{\sigma}}_{n+1}, t_{n+1} ; \overrightarrow{\boldsymbol{\sigma}}_{n}, t_{n}\right) \\
& =\lim _{N \rightarrow \infty} \int \prod_{m=1}^{N} d^{3} \boldsymbol{\sigma}_{m} \prod_{n=N}^{0} \operatorname{Exp}_{C}\left(\mathrm{H}_{S}\left(\overrightarrow{\boldsymbol{\sigma}}_{n+1}\right)\left(t_{n+1}-t_{n}\right)\right) *_{C} \delta^{3}\left(\overrightarrow{\boldsymbol{\sigma}}_{n+1}-\overrightarrow{\boldsymbol{\sigma}}_{n}\right)
\end{aligned}
$$

where $\overrightarrow{\boldsymbol{\sigma}}_{N+1}=\overrightarrow{\boldsymbol{\sigma}}_{t}$ and $\prod_{n=N}^{0}$ means that the order of the factors is inverted to maintain the leading sign. (17.28) can then be reobtained by using again the integral form of the star product to evaluate the expression and with an analogous substitution of the integrand and comparison with (7.16) and (7.26).

\section{Conclusions}

In this paper we have given an overview of several formalisms that describe the spin with Grassmann variables. All these formulations essentially lead back to the same algebra, namely the geometric algebra $\mathcal{C} \ell_{3}^{+}(\mathbb{C})$ or alternatively $\mathcal{C} \ell_{3}(\mathbb{R})$. To allow for a meaningful geometric interpretation in the non-relativistic case at hand, one of these two algebras with three Grassmann variables should be used as in this case space is three-dimensional. The remaining issue is whether the real algebra or the complex algebra is the better choice. The real algebra is, at first, the obvious choice because it offers the most elegant and plausible description of the spin whereas the complex geometric algebra naturally appears in connection with the bosonic star product formalism. It remains to be seen which of the formulations is better suited when extended to the relativistic case using for example the spacetime algebra or the algebra of physical space and when applied to specific problems. 


\section{References}

[1] F. Bayen, M. Flato, C. Fronsdal, A. Lichnerowicz and D. Sternheimer, Ann. Phys. 111 (1978) 61, 111.

[2] C. Zachos, Int. J. Mod. Phys. A 17 (2002) 297.

[3] F. A. Berezin and M. S. Marinov, Ann. Phys. 104 (1977) 336.

[4] A. C. Hirshfeld and P. Henselder, Ann. Phys. 302 (2002) 59.

[5] I. Galaviz, H. García-Compeán, M. Przanowski, F. J. Turrubiates, Weyl-Wigner-Moyal Formalism for Fermi Classical Systems, hep-th/0612245.

[6] P. Henselder, A. C. Hirshfeld and T. Spernat, Ann. Phys. 317 (2005) 107.

[7] D. Hestenes, Space-Time Algebra, Gordon and Breach, New York, 1966.

[8] D. Hestenes and G. Sobczyk, Clifford Algebra to Geometric Calculus, D. Reidel Publishing Company, Dordrecht/Boston, 1984.

[9] C. Doran and A. Lasenby, Geometric Algebra for Physicists, Cambridge University Press, Cambridge UK, 2003.

[10] A. Lasenby, C. Doran and S. Gull, J. Math. Phys. 34 (1993) 3683.

[11] E. S. Fradkin and V. Ya. Linetsky, Mod. Phys. Lett. A 6 (1991) 217.

[12] P. Henselder, Phys. Lett. A 363 (2007) 378.

[13] L. Cohen, J. Math. Phys. 17 (1976) 597.

[14] P. Sharan, Phys. Rev. D 20 (1979) 414.

[15] G. Baker, Phys. Rev. 109 (1958) 2198.

[16] R. Casalbuoni, Nuovo Cim. A 33 (1976) 389.

[17] M. A. J. Ashdown, S. S. Somaroo, S. F. Gull, J. L. Doran and A. N. Lasenby, J. Math. Phys. 39 (1998) 1566.

[18] M. R. Francis and A. Kosowsky, Ann. Phys. 317 (2005) 383.

[19] B. Demircioğlu and A. Verçin, Ann. Phys. 305 (2003) 1.

[20] A. C. Hirshfeld, P. Henselder and T. Spernat, Ann. Phys. 314 (2004) 75.

[21] D. Mauro, Phys. Lett. B 597 (2004) 94.

[22] J. A. López and J. Stephany, Spin observables and path integrals in Proceedings of the VI International Wigner Symposium (2002) 781-789. 